S77

\title{
COMPLEMENTARY ANALYTICAL APPROACHES IMPROVING KNOWLEDGE ON LACTIC ACID BACTERIA CRYORESISTANCE
}

Amelie Girardeau ${ }^{1, *}$, Julie Meneghel ${ }^{2}$, Ioan-Cristian Trelea ${ }^{1}$, Stephanie Passot ${ }^{1}$, Fernanda Fonseca ${ }^{1}{ }^{1}$ UMR GMPA, AgroParisTech, INRA, University Paris-Saclay, Thiverval-Grignon, France, France; ${ }^{2}$ Asymptote Ltd, General Electric Healthcare, Cambridge, UK, United Kingdom

* Corresponding Author.

E-mail address: amelie.girardeau@inra.fr (A. Girardeau).

Freezing is the most used bacterial cell preservation technique yet still a process that can be damaging and lead to cell death. Cryosensitivity greatly varies depending on considered species or strains. Fourier Transform InfraRed (FTIR) spectroscopy is a powerful technique allowing biochemical characterization of major cellular components (lipids, proteins, polysaccharides). However, the exploitation of bacterial spectral features under native aqueous environments is challenging due to the strong absorption of water in the mid-IR region. The identification of cryoresistance markers has thus so far been mainly done using FTIR spectra of dried cells. In this study, three lactic acid bacteria strains were selected for their contrasting cryosensitivities. The objective of this study was to combine complementary approaches to characterize bacterial cells by determining: (i) the fatty acid composition; (ii) the membrane lipid phase transition by FTIR spectroscopy combined with a temperature controlled stage and (iii) the biochemical composition of cells with a novel FTIR approach enabling higher spatial resolution in an aqueous environment. The most cryoresistant strain displayed a strikingly different membrane lipid phase transition compared to the other two: a very low lipid transition temperature $\left(-14^{\circ} \mathrm{C}\right)$, high lipid cooperativity and the maintenance of a higher degree of disorganization in the frozen state. These observations were 
ascribed to a very high unsaturated fatty acid content (C18:1, > 66\%). In addition to confirming the lipid membrane's role in cryoresistance, this study has brought to light the potential role of other cellular components such as proteins and cell wall polysaccharides. Good discrimination of strains and visualization of population heterogeneity was achieved, indicating that markers outside the lipid region certainly contribute to cryoresistance.

Source of Funding: French National Institute for Agricultural Research INRA, France

Conflict of Interest: None to disclose

\section{CHARACTERIZING HYDROGEN-BONDING CRYOPROTECTANT MIXTURES}

Kwang-Im Oh, Carlos Baiz*. University of Texas at Austin, United States * Corresponding Author.

E-mail address: cbaiz@cm.utexas.edu (C. Baiz).

Cryoprotectant agents (CPAs) are mixtures of small molecules that disrupt
ICESTART $^{\text {TM }}$ ENHANCES CRYOPRESERVATION

OF MAMMALIAN CELLS IN MULTIWELL PLATES

Martin Daily ${ }^{1, *}$, Thomas Whale ${ }^{1}$, Peter Kilbride ${ }^{2}$, Stephen Lamb $^{2}$, Benjamin Murray ${ }^{1}$, Helen Picton ${ }^{1}$, John Morris ${ }^{2} .{ }^{1}$ University of Leeds, United Kingdom; ${ }^{2}$ Asymptote, General Electric Healthcare, United Kingdom

* Corresponding Author 